\title{
Efficient Singlet Fission and Triplet-Pair Emission in a Family of Zethrene Diradicaloids
}

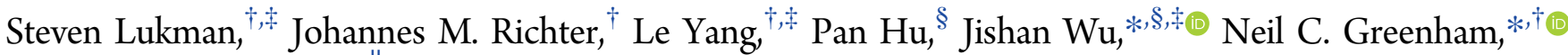 \\ and Andrew J. Musser*, $\|_{\odot}$ \\ ${ }^{\dagger}$ Cavendish Laboratory, University of Cambridge, Cambridge CB3 0HE, United Kingdom \\ ${ }^{\ddagger}$ Institute of Materials Research and Engineering, Agency for Science Technology and Research (A*STAR), 2 Fusionoplis Way, \\ Singapore 138634, Singapore \\ ${ }^{\S}$ Department of Chemistry, National University of Singapore, 3 Science Drive 3, Singapore 117543, Singapore \\ "Department of Physics \& Astronomy, University of Sheffield, Sheffield S3 7RH, United Kingdom
}

\section{Supporting Information}

ABSTRACT: Singlet fission offers the potential to overcome thermodynamic limits in solar cells by converting the energy of a single absorbed photon into two distinct triplet excitons. However, progress is limited by the small family of suitable materials, and new chromophore design principles are needed. Here, we experimentally vindicate the design concept of diradical stabilization in a tunable family of functionalized zethrenes. All molecules in the series exhibit rapid formation of a bound, spin-entangled triplet-pair state TT. It can be dissociated by thermally activated triplet hopping and exhibits surprisingly strong emission for an optically "dark" state, further enhanced with increasing diradical character. We find that the TT excited-state absorption spectral shape correlates with the binding energy between constituent triplets, providing a new tool to understand this unusual state. Our results reveal a versatile new family of tunable materials with excellent optical and photochemical properties for exploitation in singlet fission devices.

\section{INTRODUCTION}

Singlet fission (SF) is an exciton multiplication phenomenon in organic semiconductors where one spin-singlet exciton transforms into two spin-triplet excitons, conserving $\operatorname{spin}^{1,2}$ in a process that can be ultrafast $(<100 \mathrm{fs})^{3-6}$ and highly efficient. ${ }^{3,7-10}$ These characteristics and long triplet lifetimes make SF an attractive route to increase solar cell efficiencies beyond the Shockley-Queisser limit. ${ }^{11}$ Reports of successful SF-enhanced solar cells remain limited, ${ }^{12-15}$ due in part to the narrow set of known SF chromophores. Notwithstanding recent advances in polyenes, ${ }^{8,16-18}$ rylene dyes, ${ }^{19,20}$ and other small molecules ${ }^{21-24}$ most studies continue to focus on the canonical acene molecules. $^{3-5,7,25-30}$ While these materials can exhibit extremely high triplet generation efficiencies, eventual application is limited by low extinction coefficients and poor photo/chemical stability. ${ }^{31}$ Moreover, optimizing SF-based photovoltaics will require flexible choice of band gap, HOMO/LUMO levels, morphological properties, and mobility. There is thus great need to explore new chromophore design principles, with the aim of developing a wider library of SF sensitizers.

One promising design motif with wide scope for tunability is diradical stabilization. ${ }^{1,32,33}$ A diradical molecule has a pair of approximately degenerate orbitals occupied by two electrons in

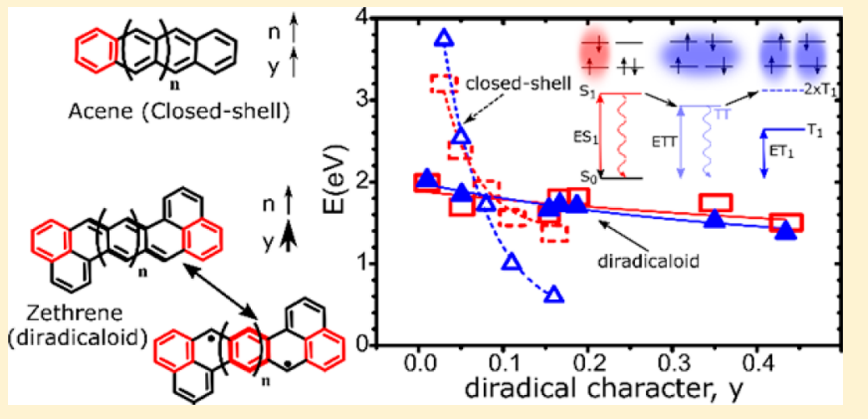

the ground state, yielding minimal separation between lowest energy singlet and triplet states. Structural perturbation lifts the degeneracy to form a diradicaloid, with double occupation of the more stable orbital reducing the energy of $S_{0}$ and increasing those of $T_{1}$ and $S_{1}$. Sufficiently strong perturbation can fulfill the primary SF energetic condition $\Delta E_{\mathrm{SF}}=2 \times E\left(\mathrm{~T}_{1}\right)-E\left(\mathrm{~S}_{1}\right)$ $\leq 0$. This principle was demonstrated by the observation of SF in 1,3-diphenylisobenzofuran. ${ }^{10}$ However, it has not been further applied, and the proposed correlations ${ }^{32-34}$ between molecular structure, diradical character, and energy levels have yet to be verified experimentally.

Here, we systematically explore the photophysics of a family of zethrene diradicaloids, ${ }^{35-37}$ which exhibit quinoidal and diradical resonance forms as shown in Figure 1a. The aromatic sextet rings (red) in the diradical structure offset the energetic cost of radical formation and increase the overall diradical contribution. The diradical character ensures small triplet energies and strong two-photon absorption, ${ }^{35,38,39}$ while the quinoidal core of the more stable resonance structure affords a high absorption coefficient. ${ }^{40}$ This combination holds great promise for applications, ${ }^{41}$ and recent strategies to chemically

Received: October 12, 2017

Published: November 22, 2017 


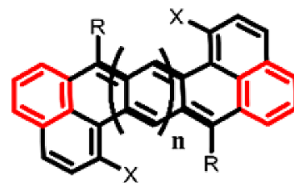

Zethrene, $\mathrm{Z}(\mathrm{n}=0)$

Heptazethrene, $\mathrm{HZ}(\mathrm{n}=1)$

Octazethrene, $\mathrm{OZ}(\mathrm{n}=2)$

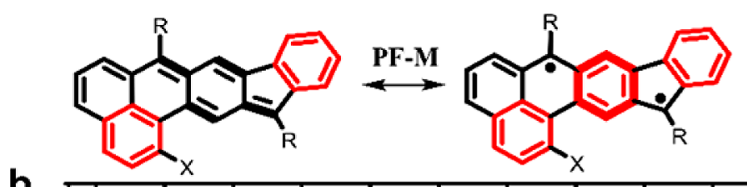

b

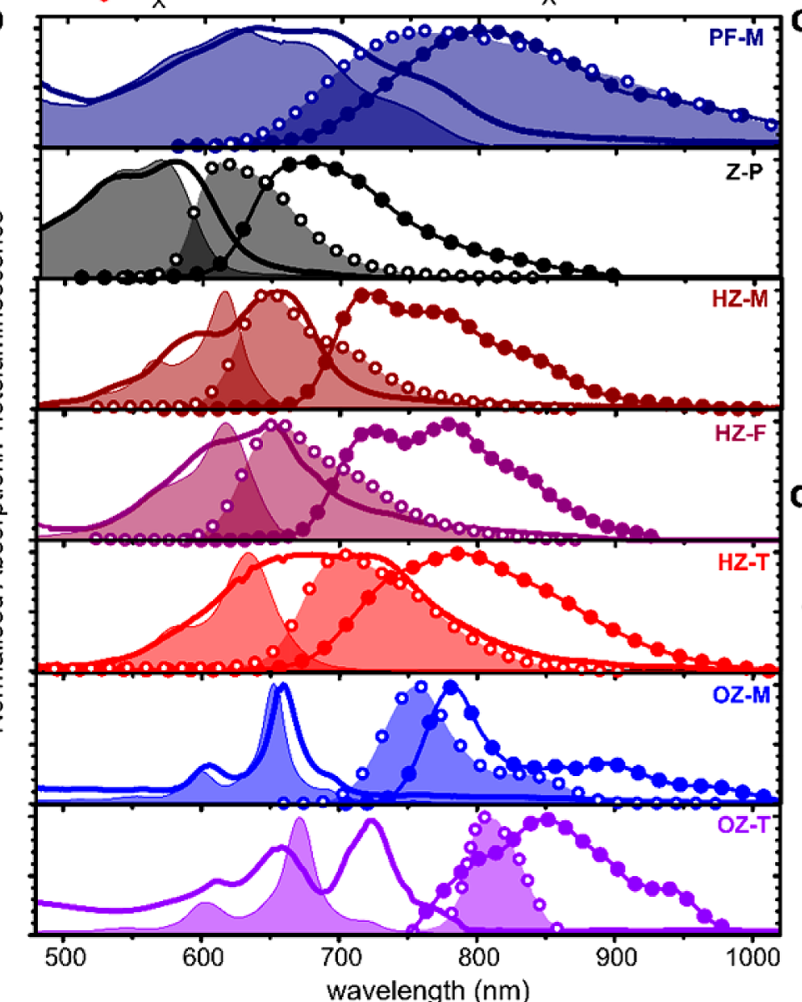

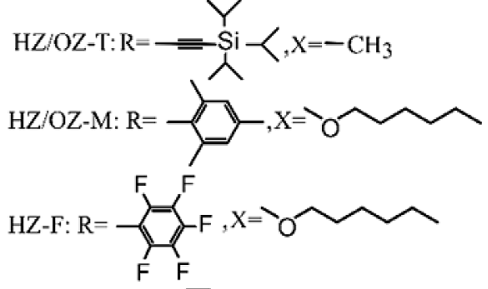

$\mathrm{Z}-\mathrm{P}: \mathrm{R}=\longrightarrow \mathrm{Z}, \mathrm{X}=\mathrm{H}$

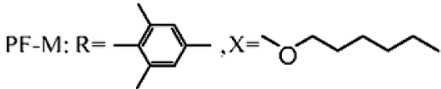

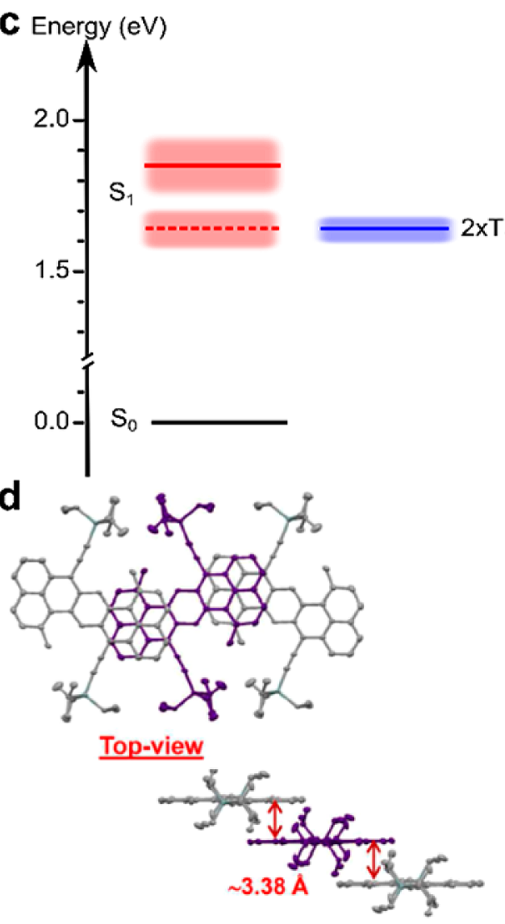

$\underline{\text { Side-view }}$

Figure 1. Steady-state characterization. (a) Molecular structures of zethrene diradicaloids used in this study. (b) Ground-state absorption and photoluminescence spectra of chloroform solutions (filled) and thin films (solid lines). (c) Energy level diagram of HZ-T. E( $\left.\mathrm{S}_{1}\right)$ in solution (solid) and film (dashed) and $2 \times E\left(\mathrm{~T}_{1}\right)$ are determined from fluorescence and phosphorescence. (d) Top and side view of HZ-T packing structures. Hydrogen atoms omitted for clarity.

protect the reactive radical sites have opened a library of tunable, highly stable derivatives. ${ }^{35-39}$ We investigated the smallest member of the class, six-ring zethrene $(\mathrm{Z})$, larger heptazethrene $(\mathrm{HZ})$ and octazethrene $(\mathrm{OZ})$, and phenalenofluorene $(\mathrm{PF})$. We demonstrate their general ability to undergo rapid $S F$, via the same bound, spin-entangled, and surprisingly emissive triplet-pair state TT recently reported in acenes.,15 This state is stabilized with respect to two free triplets $(\mathrm{T}+\mathrm{T})$ and enables efficient SF even against nominally unfavorable energetics $\left(\Delta E_{\mathrm{SF}} \geq 0\right)$. It subsequently separates into $\mathrm{T}+\mathrm{T}$ through thermally activated triplet hopping. The rates of both processes are governed by the state energies-controlled by diradical character-and intermolecular packing.

\section{METHODS}

Materials. Z-P, HZ-T, HZ-M, HZ-F, OZ-M, OZ-T, and PF-M were synthesized as previously reported. ${ }^{35,37-39}$ Details of synthesis, NMR characterization, and X-ray crystallographic characterization can be found in previous publications.
Sample Preparation. The solubilizing side groups on zethrene derivatives provide chemical stability and render significant solubility. Unless otherwise stated, all measurements in dilute solutions were performed at $0.1 \mathrm{mM}$ in chloroform, prepared and sealed under nitrogen atmosphere. High-concentration solutions were prepared at 8 $\mathrm{mM}$ in chloroform. Triplet sensitization was performed using established procedures ${ }^{18}$ with a mixed solution in chloroform of 0.5 $\mathrm{mg} / \mathrm{mL}$ of the molecule of interest and $1.5 \mathrm{mg} / \mathrm{mL} \mathrm{N}$-methylfulleropyrrolidine. All solution measurements were performed in sealed quartz cuvettes with $1 \mathrm{~mm}$ light path, except high-concentration solutions in which a $0.1 \mathrm{~mm}$ light path was used (Hellma Analytics). To prepare thin films, $5 \mathrm{mg} / \mathrm{mL}$ solutions in chloroform were spun on Spectrosil at $800-1200 \mathrm{rpm}$ for $40-60 \mathrm{~s}$ and then annealed at $60-80$ ${ }^{\circ} \mathrm{C}$ for 30-60 min. To obtain samples that gave phosphorescence from individual, isolated chromophores, the molecules were mixed with platinum octaethylporphyrin (PtOEP, purchased from Sigma-Aldrich) and dispersed in polystyrene matrix. The final weight percentage of molecule:PtOEP:polymer in the mixture was 1:5:94. This mixture was drop casted on Spectrosil. Thin film phosphorescence samples were prepared in a similar manner, in which the target molecule:PtOEP ratio was varied from 95:5 to $98: 2$ wt \% to give the clearest signal. 
Table 1. Diradical Optical Properties and Energy Levels Relevant to Singlet Fission

\begin{tabular}{|c|c|c|c|c|c|c|c|c|c|}
\hline & & $\varepsilon_{\max }\left(\operatorname{lmol}^{-1} \mathrm{~cm}^{-1}\right)$ & $\tau_{\mathrm{s}}(\mathrm{ps})^{a}$ & PLQE (\%) & $y^{b}$ & $E\left(\mathrm{~S}_{1}\right)$ abs. $(\mathrm{eV})$ & $E\left(\mathrm{~S}_{1}\right) \mathrm{PL}(\mathrm{eV})$ & $E(\mathrm{TT})_{0-1}\left(E\left(\mathrm{TT}_{0-0}\right)\right)(\mathrm{eV})$ & $E\left(\mathrm{~T}_{1}\right)-E\left(\mathrm{~S}_{0}\right)(\mathrm{eV})^{c}$ \\
\hline \multirow[t]{2}{*}{ Z-P } & sol & 35500 & 4400 & 72 & $0.01^{d}$ & 2.19 & 2.03 & & 1.01 \\
\hline & film & & 1.25 & 11 & & 2.02 & 1.88 & $1.73(1.85)$ & 1.00 \\
\hline \multirow[t]{2}{*}{ PF-M } & sol & 6760 & 7.20 & $<1$ & 0.051 & 1.85 & 1.73 & & 0.93 \\
\hline & film & & 1.81 & 14 & & 1.81 & 1.69 & $1.59(1.69)$ & 0.92 \\
\hline \multirow[t]{2}{*}{$\mathrm{HZ}-\mathrm{M}$} & sol & 70030 & 5500 & 67 & 0.167 & 2.02 & 1.91 & & 0.85 \\
\hline & film & & 0.25 & $<1$ & & 1.84 & 1.73 & $1.53(1.65)$ & 0.84 \\
\hline \multirow[t]{2}{*}{ HZ-F } & sol & 42180 & 5100 & 65 & 0.167 & 2.01 & 1.89 & & 0.86 \\
\hline & film & & 0.26 & $<1$ & & 1.82 & 1.72 & $1.57(1.69)$ & 0.89 \\
\hline \multirow[t]{2}{*}{ HZ-T } & sol & 60000 & 3400 & 69 & 0.154 & 1.95 & 1.76 & & 0.83 \\
\hline & film & & 0.21 & $<1$ & & 1.70 & 1.66 & $1.51(1.64)$ & 0.82 \\
\hline \multirow[t]{2}{*}{$\mathrm{OZ}-\mathrm{M}$} & sol & 87000 & 2300 & 59 & 0.35 & 1.83 & 1.64 & & 0.76 \\
\hline & film & & 0.16 & $<1$ & & 1.72 & 1.59 & $1.36(1.48)$ & 0.79 \\
\hline \multirow[t]{2}{*}{ OZ-T } & sol & 83300 & 1600 & 62 & 0.434 & 1.84 & 1.53 & & 0.69 \\
\hline & film & & 0.18 & $<1$ & & 1.55 & 1.43 & $1.22(1.32)$ & 0.69 \\
\hline
\end{tabular}

${ }^{a}$ Singlet lifetime in solution and film is an average between exponential-fitted lifetime from PL and TA measurements. ${ }^{b}$ Adopted from refs 33 , 36, and 37. ${ }^{c}$ Measured with $\mathrm{Pt}$-porphyrin-sensitized phosphorescence. ${ }^{d} \mathrm{~A} y$ value for Z-P has not been reported using a comparable calculation method to the other materials, but it is known to be small (although not negligible); hence, we tentatively assign it as 0.01 .

Mixtures were spin coated on Spectrosil at 800-1200 rpm for 40-60 s in a nitrogen-filled glovebox and then annealed at $60-80{ }^{\circ} \mathrm{C}$ for $30-$ $45 \mathrm{~min}$. All films were measured under vacuum or sealed between quartz slides with epoxy under nitrogen atmosphere to prevent degradation and triplet quenching by oxygen.

Spectroscopic Measurements. UV-vis absorption spectra were measured with a Cary $400 \mathrm{UV}$-vis spectrometer. Steady-state PL spectra were measured using a calibrated infrared InGaAs photodiode array (ANDOR iDus 490A) coupled to a spectrograph (ANDOR Shamrock). The PL dynamics were measured in two distinct temporal regimes. Fast (sub-100 ps) photoluminescence dynamics were studied using a photoluminescence upconversion technique (setup detail in Supporting Information). The PL dynamics at early time were also measured with a transient grating technique with a home-built TGPL spectrometer (details can be found in Supporting Information), which can only cover up to $750 \mathrm{~nm}$. The longer time dynamics were recorded with a standard time-correlated single-photon counting (TCSPC) system. Supplementary Information gives more details on the TCSPC setup. Phosphorescence was detected using a calibrated infrared InGaAs photodiode array (ANDOR iDus 490A) coupled to a spectrograph (ANDOR Shamrock) with CW excitation at $532 \mathrm{~nm}$ $(0.65 \mathrm{~mW})$. The PL quantum efficiency was determined on the same system versus Nile Blue reference. Details on the transient absorption setup can be found in the Supporting Information. All thin film results were also carefully checked for the presence of artifacts due to thermal effects from laser excitation, which result from small changes in film absorption from laser heating. These are known to result in derivativelike lineshapes in the region of GSB, with the same linear dependence on pump intensity observed in excitonic signals and long lifetimes comparable to triplets or polarons. The spectral effects were estimated by recording thin-film absorption spectra under heating. The hightemperature absorption spectra were subtracted from room-temperature spectra, and this $\Delta \mathrm{OD}$ signal was converted to $\Delta T / T$ to generate the expected TA thermal artifact.

Spectral Decomposition. Singular value decomposition (SVD) was used to determine the number of components/species in transient absorption and PL measurements. A component is considered real and representative of a unique spectral species if the singular value (eigenvalue) is higher than the baseline noise level (Supplementary Figure 9). The associated spectra themselves are not representative of electronic species but rather linear combinations of them. All TA data plotted in the main text and Supporting Information had been removed from random noise by applying SVD. The spectral shapes and time evolution of these species were optimized by a genetic algorithm operating on the full TA data set. The advantage of this approach is that it starts from randomized initial spectra and does not require a predetermined model or kinetic scheme; the only input is the number of spectrally distinct species present in the data matrix. The genetic algorithm is suitable to model multidimensional data and less likely to be trapped in local minima. Indication of a good data fit is good correlation with reference spectra and reproducibility. For subpicosecond TA and PL upconversion measurements, the algorithm was performed between the instrument-limited time resolutions $(\sim 100-200 \mathrm{fs})$ up to $2 \mathrm{~ns}$ (TA) or 100 ps (PL up-conversion). For longer time (ns) measurements, the code was run from 3 to $100 \mathrm{~s} n$ s. We further confirm the reliability of the results by comparing the optimized spectra and kinetics to the raw data. Close agreements between spectral shapes and kinetics between four independent measurements (short- and long-delay TA, PL upconversion, and TCSPC) ensure that the results are robust.

Diradical Character. The diradical index was taken from literature sources, and the values were calculated on the basis of the LUMO occupation number $(n)$ in natural orbital analysis, for the UCAMB3LYP/6-31G(d,p $)^{42-44}$ optimized open-shell singlet geometry. $y$ is formally expressed as $y=1-(2 \mathrm{~T} /(1+\mathrm{T} 2))$, where $\mathrm{T}$ is represented by using the occupation numbers of natural orbitals as $\mathrm{T}=(n \mathrm{HOMO}$ - $n$ LUMO) $/ 2 .^{33,45}$ A molecule with $y=0$ indicates a closed-shell structure, whereas a molecule with $y=1$ implies a pure diradical structure. Any intermediate value of $y$ refers to diradicaloid (i.e., diradical-like) structures.

\section{RESULTS AND DISCUSSION}

Diradical character is ranked with quantum-chemical index $y$, a measure of effective bond weakness or electron correlation. This gauge of electron localization on the radical sites ranges from completely delocalized (0) to localized (1). ${ }^{46}$ The seven diradicaloids studied here exhibit substantial variation in $y$ (Table 1), showing sensitivity to the number and type of conjugated rings and side groups. . $^{35,36,38,39}$ The latter are also crucial for molecular packing, and we have explored their effect with phenylethynyl (P), triisopropylsilylethynyl (T), mesityl (M), and fluorinated mesityl (F) substituents. Steady-state absorption and fluorescence (Figure 1b, Table 1) and sensitized phosphorescence (Supplementary Figure 1) confirm that all molecules except PF-M fulfill $\Delta E_{\mathrm{SF}} \leq 0$ in solution, and all but Z-P, PF-M, and HZ-F do so in thin film. The endothermicity of the latter systems $(\sim 60-150 \mathrm{meV})$ is nonetheless comparable to tetracene, ${ }^{1}$ and SF may still be possible. We note that the observed phosphorescence contrasts with energies estimated from temperature-dependent SQUID measurements, ${ }^{35,38}$ which generally suggest $E\left(\mathrm{~T}_{1}\right)<\sim 200 \mathrm{meV}$. The long triplet lifetimes 
a
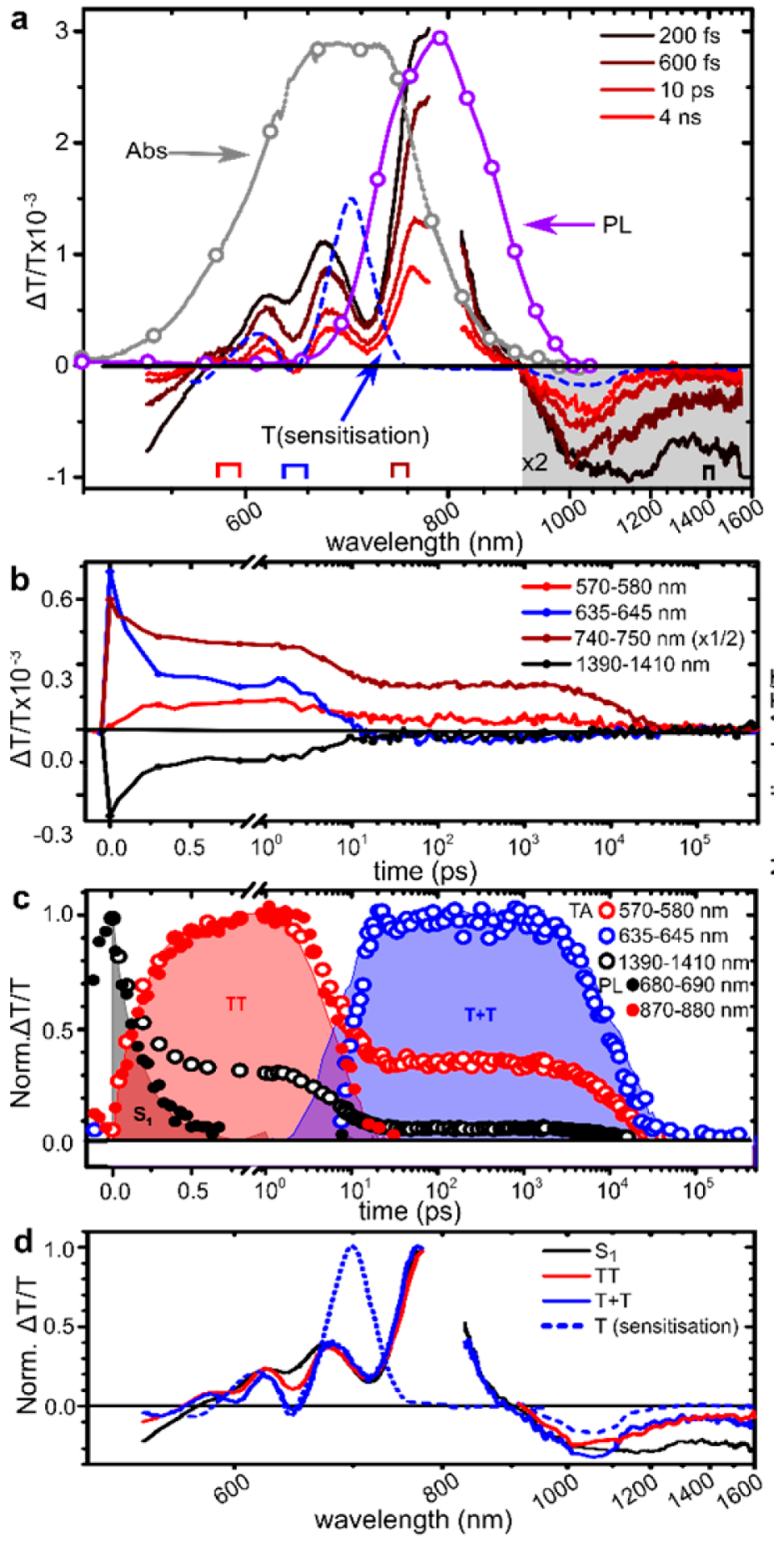

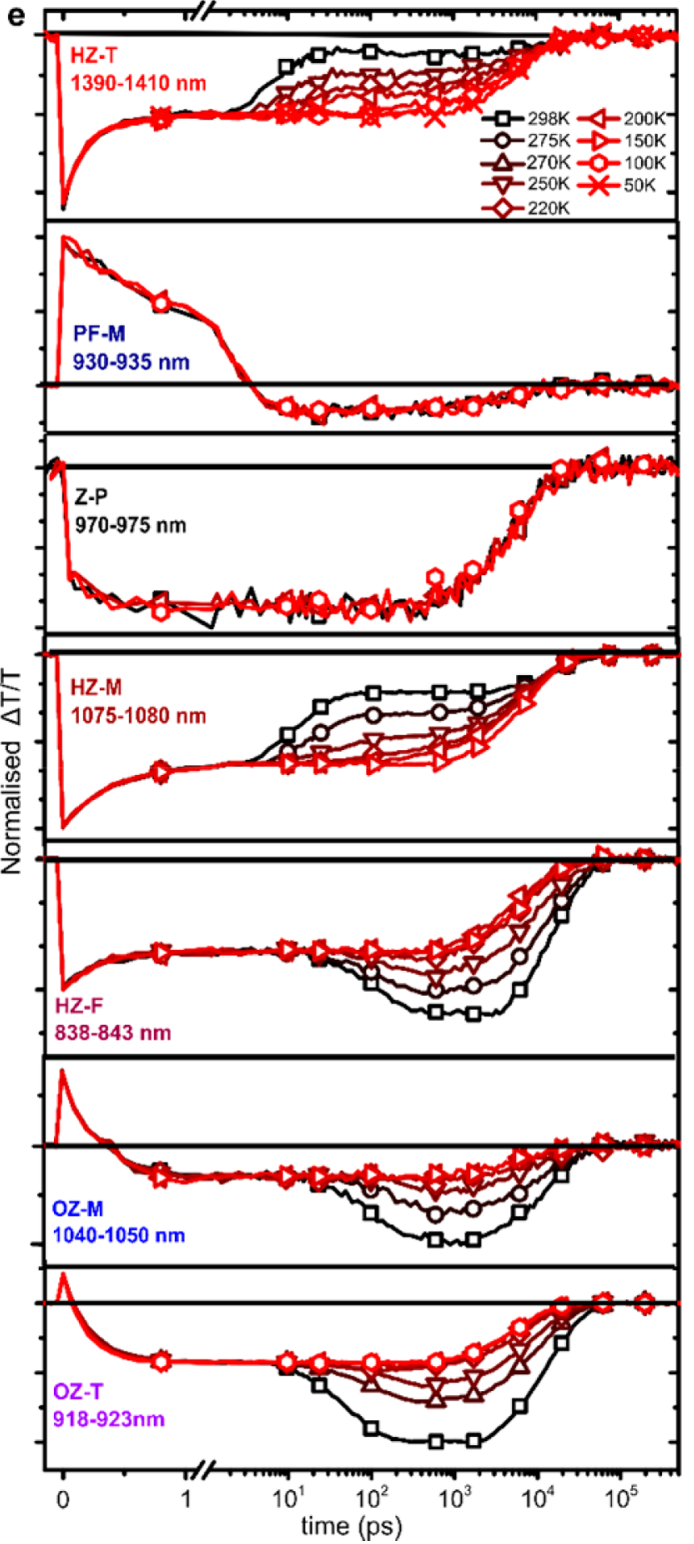

Figure 2. Singlet fission via TT intermediate. (a) Transient absorption spectra of HZ-T thin film at $298 \mathrm{~K}$ at the indicated pump-probe delays. Steady-state absorption (gray circles) and PL (purple circles) are presented for reference. Excited-state absorption signatures of terminal state from $10 \mathrm{ps}$ to $4 \mathrm{~ns}$ closely resemble the triplet from solution sensitization (blue dashed). Brackets highlight regions integrated for kinetics. (b) Corresponding TA kinetics in the bands indicated, showing two distinct temporal regimes: fission of $S_{1}$ into correlated triplet pair TT ( 200 fs) and separation of TT into free T+T ( $\sim 6 \mathrm{ps})$. (c) Normalized population kinetics of excited-state species extracted from TA maps at $298 \mathrm{~K}$, along with integrated raw TA (circles) and PL kinetics (dots). (d) Species-associated spectra of states relevant to SF in HZ-T. (e) Temperature-dependent TA kinetics revealing invariant $S_{1} \rightarrow$ TT conversion and thermal activation of TT $\rightarrow$ T+T separation in HZ-T (top) and all other molecules. PF-M and Z-P are fully temperature independent. All traces capture a mixture of spectral signatures: $\mathrm{S}_{1}$ PIA or SE immediately following photoexcitation, TT PIA dominating from $\sim 1$ to $5 \mathrm{ps}$, and, in all materials except PF-M and Z-P, T+T dominating from $\sim 100$ ps at high temperatures (black). Kinetics are normalized to initial signal. Full data presented in Supplementary Figures 10-30.

we measure in solution sensitization experiments (Supplementary Figures 2 and 4-8) and porphyrin-sensitized phosphorescence spectra cannot be reconciled with such low triplet energies. Our comprehensive spectroscopic study here can only be rationalized in terms of these markedly higher triplet energies, measured with the most direct method available, and we suggest that the earlier measurements reflected charged species rather than excitonic triplets, as suggested by ESR measurements. ${ }^{47}$ Most of the molecules studied exhibit moderately high solution photoluminescence quantum efficiency (PLQE, Table 1), indicating that there are no strong competing decay pathways for the initially photoexcited state such as internal conversion into low-lying "dark" states - a second requirement for an effective SF sensitizer. Indeed, transient absorption measurements in solution reveal concentration-dependent conversion of singlets into two triplet excitons, a hallmark of diffusive SF $^{25,26,48,49}$ (Supplementary Figures 2 and $4-8$ ). The only exception is PF-M, which has an extremely short excited-state lifetime pointing to efficient nonradiative decay (Table 1, Supplementary Figure 3).

Steady-State Optical Characterization. The groundstate absorption spectra in Figure la reveal a systematic 

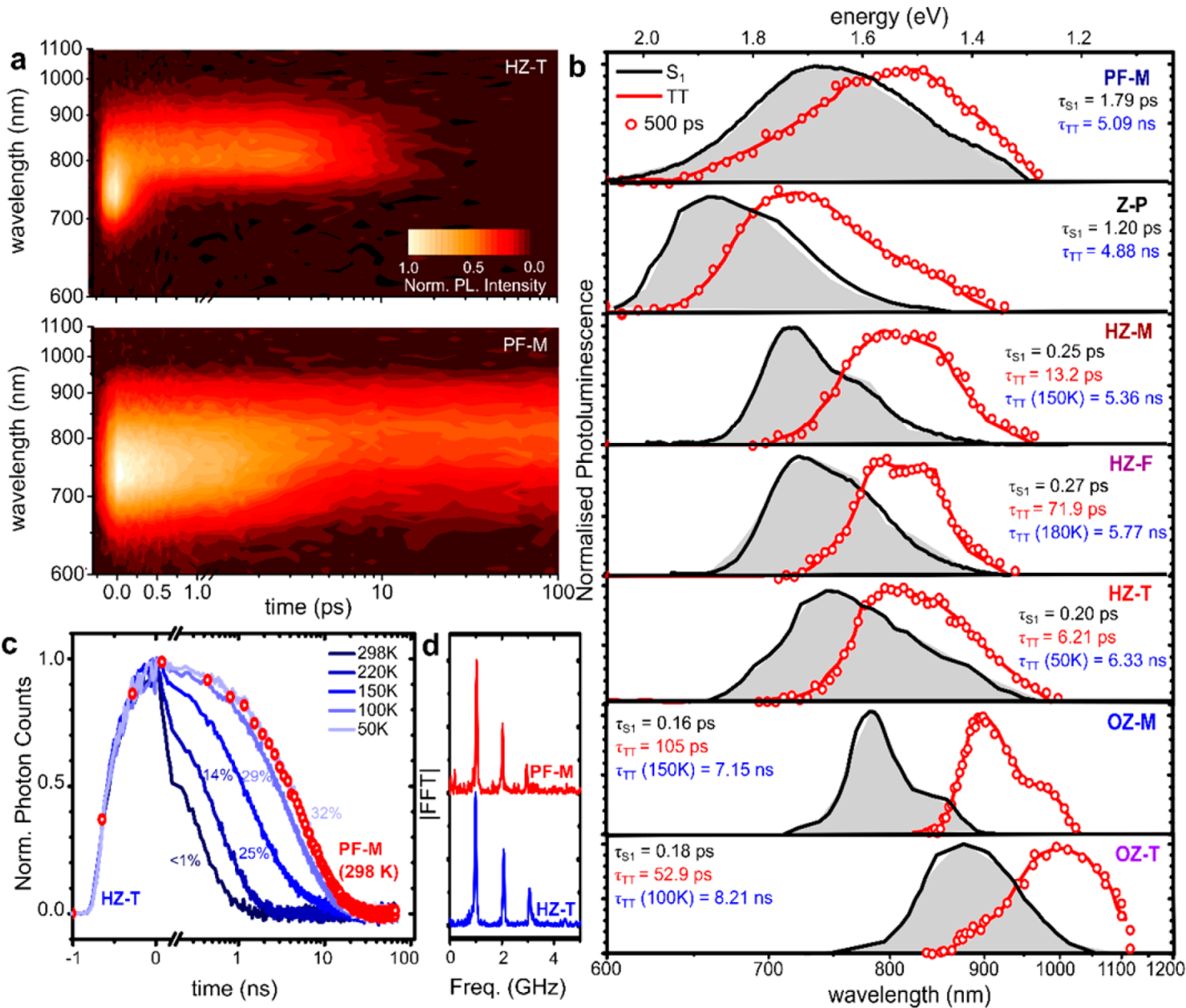

Figure 3. Direct TT emission. (a) Subpicosecond PL maps of HZ-T (top) and PF-M (bottom) films at $298 \mathrm{~K}$ following $<200 \mathrm{fs}$ excitation. Initial $\mathrm{S}_{1}$ emission decays rapidly, giving rise to lower energy emission assigned to TT. (b) Decomposition of PL maps yields distinct $\mathrm{S}_{1}$ and TT species (lines) for all molecules. PL lifetimes of $S_{1}$ (black), TT at $298 \mathrm{~K}$ (red), and TT at low temperature (blue) closely match TA kinetics for these species. Lowtemperature data reflects intrinsic TT lifetime, when $\mathrm{T}+\mathrm{T}$ formation is fully suppressed. TCSPC wavelength scan (circles) reveals only trace TT emission on long time scales. Shaded $S_{1}$ spectra obtained by subtracting the TT spectrum from steady-state PL. (c) Temperature-dependent TT PL kinetics in HZ-T film, measured with TCSPC. Lifetime and quantum yield (indicated) increase upon cooling, in accord with TA kinetics. TT emission in PF-M (red circles) is temperature invariant, and only $298 \mathrm{~K}$ data are plotted. (d) Fast Fourier transform (FFT) of residual HZ-T (100 $\mathrm{K}$ ) and PF-M (298 K) PL oscillations, following subtraction of exponential decay from TCSPC kinetic. Well-defined quantum beats at $0.99,2.08$, and $3.09 \pm 0.1 \mathrm{GHz}(\mathrm{HZ}-\mathrm{T})$ and 1.03, 2.02, and $2.95 \pm 0.1 \mathrm{GHz}(\mathrm{PF}-\mathrm{M})$ demonstrate $\mathrm{SF}$ in both films, even though no T+T is formed. Equivalent PL upconversion and temperature-dependent TCSPC data for all materials presented in Supplementary Figures 10, 12, 15, 18, 25 , and 28.

decrease in the optical gap with increasing molecular size. The effect is markedly smaller than in acenes, due to the predisposition of zethrenes to form localized diradicals which lessen the effective conjugation. The high diradical character in $\mathrm{OZ}(y \approx 0.35-0.45)$ has also been suggested to result in a lowlying doubly excited singlet state approximately isoenergetic with the dominant transition, ${ }^{35,50}$ manifested in the weak absorption at wavelengths greater than $700 \mathrm{~nm}$. This state endows the molecule with significant two-photon absorption cross-section, observed also in HZ-T. ${ }^{35}$ However, we find no evidence of conversion between these singly and doubly excited states in the dilute-solution photophysics, where the initially photoexcited state emits efficiently and decays uniformly in 2.1-5.5 ns (Supplementary Figures 2 and 4-8). We further note the energetic effects of the side groups. Triple-bond substituents $\mathrm{P}$ and $\mathrm{T}$ distinctly enhance electron delocalization and pull down the LUMO level (i.e., HZ-M vs HZ-T). The absorption of $\mathrm{T}$ molecules becomes even more red shifted and broadens significantly in thin film. This highlights the tunability of the molecular packing: $\mathrm{T}$ side groups are less sterically demanding than $\mathrm{M}$ or $\mathrm{F}$ groups, yielding closer packing with stronger intermolecular electronic coupling. ${ }^{35,38}$

In all materials except PF-M, film formation results in sharply reduced room-temperature PLQE (Table 1). This efficient quenching signifies a new singlet decay channel, likely to be SF in light of the energetic structure. The behavior of PF-M films is surprising, exhibiting instead substantially increased PLQE. As we show below, this effect is also a consequence of SF into the emissive triplet-pair state TT. Indeed, direct emission from TT accounts for a major portion $(30-60 \%)$ of room-temperature photoluminescence in all of the films studied and becomes substantially greater at reduced temperature. To understand these effects, we apply complementary ultrafast spectroscopy techniques: transient absorption (TA), time-resolved photoluminescence upconversion, and time-correlated single-photon counting (TCSPC). These have been performed on all materials over a range of temperatures $(50-298 \mathrm{~K})$, with full data presented in Supporting Information. Here we focus 
primarily on the model zethrene HZ-T. Thin-film HZ-T has optimal SF energetics $\left(\Delta E_{\mathrm{SF}} \approx 0\right.$, Figure $\left.1 \mathrm{~b}\right)$ and packs in a slip-stacked structure with significant nearest-neighbor orbital overlap (Figure 1c), an important factor for triplet formation.

Excited-State Progression in HZ-T. We present TA data in units of differential probe transmission, $\Delta T / T$, in which the photoinduced absorption (PIA) of excited states appears negative, while positive signals indicate the bleaching of the ground state (GSB) or stimulated emission from bright excited states (SE). TA data were collected over the delay range from 100 fs to 400 ns, and the spectra in Figure 2a are dominated by GSB peaks matching the ground-state absorption (gray circles). On early time scales there is a weak additional contribution around $800 \mathrm{~nm}$ from SE, in agreement with the steady-state PL (purple circles). The primary changes in the spectral shape occur within $\sim 500$ fs: decay of SE and the PIA band $>1400 \mathrm{~nm}$, accompanied by formation of a PIA peak $\sim 1000 \mathrm{~nm}$ and GSB growth around $575 \mathrm{~nm}$. These reveal rapid internal conversion from the bright singlet state, consistent with the efficient PL quenching. A second conversion is distinguished in the kinetics (Figure 2b) from 3 to $20 \mathrm{ps}$, leaving a small PIA at $640 \mathrm{~nm}$ and a distinct PIA band shifted from $\sim 1000$ to $1040 \mathrm{~nm}$. This terminal state decays uniformly over tens of nanoseconds and matches the triplet reference spectrum from solution sensitization apart from the different underlying GSB (Figure 2a, blue dashed line). From this spectral match and the long lifetime, we assign these signatures to triplet excitons rapidly formed via SF. At high excitation density, we observe no change in the kinetics of depletion of the singlet state or in the formation of final triplet features. However, there is a clear reduction in the final triplet lifetime, an indication that the species present on these time scales is capable of diffusion and thus of bimolecular triplet-triplet annihilation (Supplementary Figure 21). On the basis of this behavior, the close match to triplet sensitization spectra in solution, and the thermal activation discussed below, we can assign this final state specifically to unbound triplet pairs $\mathrm{T}+\mathrm{T}$. Immediately following singlet decay, we observe a PIA peak at $\sim 1000 \mathrm{~nm}$ not found in the singlet or the terminal $\mathrm{T}+\mathrm{T}$ state, indicating the presence of an intermediate state. ${ }^{6,15,26,51,52}$ We isolate its characteristics using spectral decomposition, which confirms the presence of three distinct excited states (Supplementary Figure 9) and supports the sequential pathway suggested from the raw kinetics (Figure $2 \mathrm{~b}$ and $2 \mathrm{c}$ ). This intermediate spectrally resembles $\mathrm{T}+\mathrm{T}$ but with a blue shift of the PIA spectral weight $>900 \mathrm{~nm}$ (Figure $2 \mathrm{~d}$ ). Its conversion into $\mathrm{T}+\mathrm{T}$ is strongly temperature dependent and is suppressed upon cooling (Figure 2e, top). This behavior recalls the thermally activated free triplet formation recently identified in $\mathrm{F}_{2}$-TESADT and several acenes, ${ }^{6,15,27}$ and we similarly assign the intermediate to the entangled TT pair.

Our assignment is confirmed below with photoluminescence spectroscopy. The decay of TT is monoexponential at temperature extremes: 6 ps at $298 \mathrm{~K}$, with complete conversion into $\mathrm{T}+\mathrm{T}$, and $4.2 \mathrm{~ns}$ at $50 \mathrm{~K}$, where no $\mathrm{T}+\mathrm{T}$ is formed. Between these limits a variable fraction separates into $\mathrm{T}+\mathrm{T}$. In all instances, the decay of TT is completely independent of excitation density, confirming that recombination in this regime is geminate (Supplementary Figure 22).

We observe strikingly similar behavior in the other exothermic $\mathrm{HZ}$ and $\mathrm{OZ}$ materials (Figure 2e and Supplementary Figures 15, 18, 25, and 28). All exhibit temperatureindependent SF into the TT intermediate, with subsequent thermally activated separation into $\mathrm{T}+\mathrm{T}$. Interestingly, in spite of unfavorable energetics both PF-M and Z-P also exhibit rapid $(<10 \mathrm{ps})$ singlet decay into the emissive bound pair TT, characterized by blue-shifted triplet PIA bands (see below). This result demonstrates that the intertriplet binding energy is sufficient to bring $E(\mathrm{TT})$ below $E\left(\mathrm{~S}_{1}\right)$ in these endothermic materials, though the triplets consequently remain bound at all temperatures.

Direct TT Emission. Subpicosecond photoluminescence upconversion measurements offer further insight into TT. In HZ-T films, the initial slightly Stokes-shifted singlet emission is peaked at $750 \mathrm{~nm}$ (Figure 3a). This fluorescence decays completely within $500 \mathrm{fs}$, concomitant with the growth of a redshifted emission centered at $\sim 810 \mathrm{~nm}$.

The formation and decay of the red-shifted emission coincide precisely with the kinetics of TT observed in TA (compare filled and open symbols, Figure 2c). This unambiguous correlation with $\mathrm{TT}$ requires either that $\mathrm{TT}$ exists in equilibrium with some other low-energy singlet-character state which we are unable to distinguish in steady-state or transient absorption or that we are detecting direct fluorescence from TT itself. As discussed below, the energetic position of this emission tracks well the measured triplet energies, lending weight to the assignment to TT fluorescence. We detect two distinct emissive species in all seven molecules: initial $S_{1}$ and red-shifted TT, which show in every instance excellent agreement with the corresponding TA kinetics (Figure 3a and $3 \mathrm{~b}$ and Supplementary Figures 10, 15, 18, 25, and 28).

The TT emission exhibits the same temperature dependence identified in TA, measured here using TCSPC with temporal resolution $\approx 300 \mathrm{ps}$. On these time scales, the only signal arises from the delayed TT emission (circles in Figure $3 \mathrm{~b}$ ) with no detectable spectral variation upon cooling (Supplementary Figure 23). At high temperature we observe instrument-limited decay (Figure 3c). The PL lifetime and quantum yield progressively increase with cooling, leading to long-lived and surprisingly efficient TT emission (up to 32\%) at low temperature. Similar temperature-dependent behavior was observed for all exothermic materials (Figure 3b, Supplementary Figures 15, 18, 25, and 28). By contrast, endothermic PF$\mathrm{M}$ (Figure 3c and Supplementary Figure 12) and Z-P (Supplementary Figure 10) show constant TT emission lifetime and yield at all temperatures. The case of PF-M is particularly noteworthy: SF efficiently outcompetes intrinsic nonradiative decay of the singlet state, resulting in a TT state which is $>14$ times more emissive than $S_{1}$ in solution.

We additionally resolve quantum beating in the PL decay dynamics of HZ-T (100 K) and PF-M (298 K), conditions in which no free triplets are formed. This phenomenon unambiguously demonstrates $\mathrm{SF}$, as extensively studied in tetracene $^{30,53,54}$ and, recently, $\mathrm{F}_{2}$-TES-ADT. ${ }^{15}$ Following subtraction of the exponential TT decay profile from the TCSPC kinetics, the Fourier transform of the residual oscillations yields three distinct beat frequencies (Figure $3 \mathrm{~d}$ ). The origin of such beating has been explored in detail elsewhere, ${ }^{30,53,54}$ and we provide here only a brief summary. The simplest complete picture of SF requires consideration of 2 electrons and 2 holes, and these can be combined in 16 different spin configurations: 2 singlets, 9 triplets, and 5 quintets. SF entails conversion between the 2 singlet configurations " $\mathrm{S}_{1} \mathrm{~S}_{0}$ " and " $(\mathrm{TT})$ ". The latter is a superposition of triplet-pair wave functions and is not an energy eigenstate of the system, and as a result these components acquire a relative 

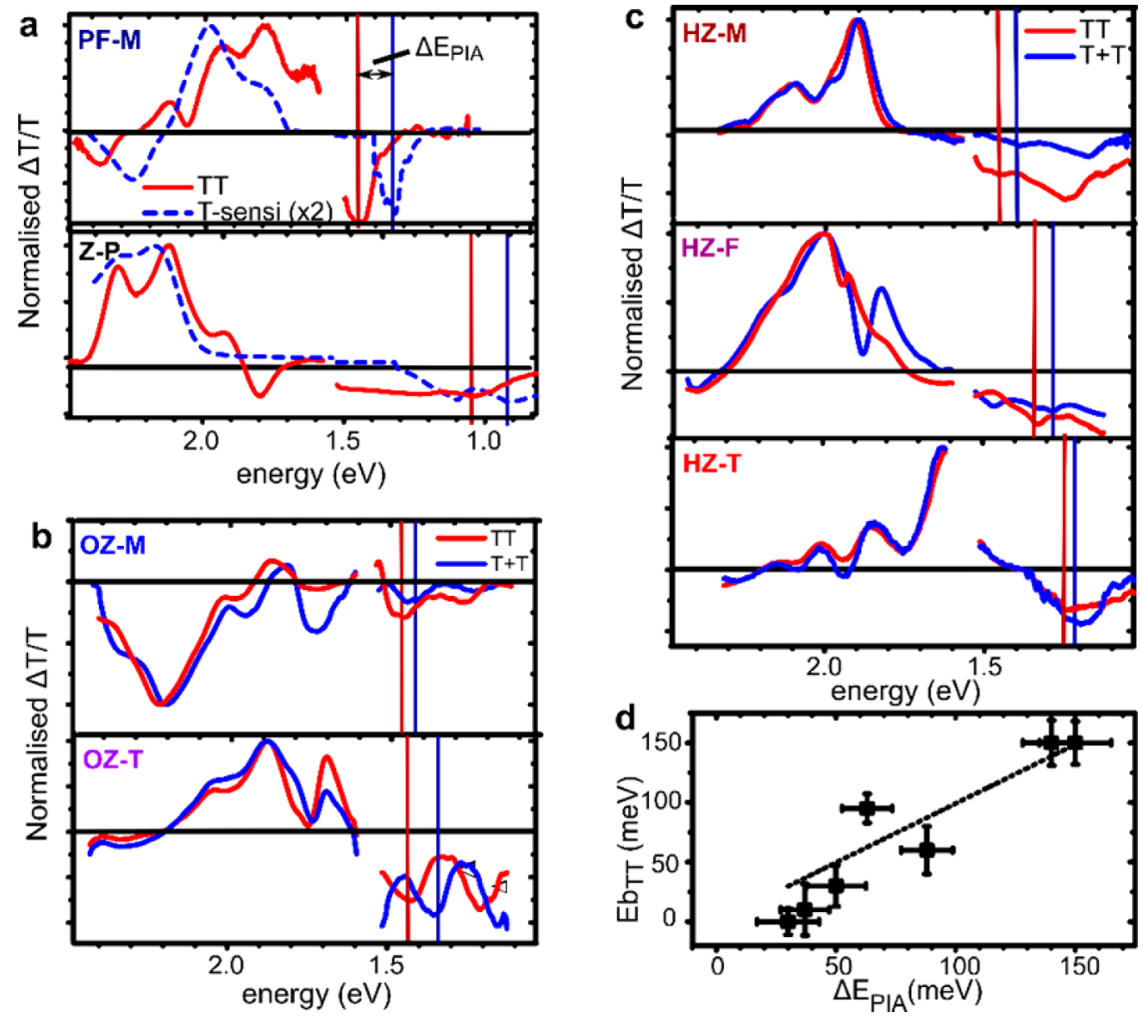

Figure 4. Spectral signatures of TT binding. Extracted TA spectral shapes of bound (TT, red) and free (T+T, blue) triplet pairs or sensitized triplets (blue dashed) for (a) PF-M and Z-P, (b) OZ family, and (c) HZ family, converted to eV scale for comparison. Data $<1.6 \mathrm{eV}$ scaled $2 \times$ for clarity. Degree of blue shift $\Delta E_{\mathrm{PIA}}$ determined from indicated equivalent $\mathrm{T}_{1} \rightarrow \mathrm{T}_{n}$ PIA peaks. (d) TT binding energy $E_{\mathrm{b}}(\mathrm{TT})$, calculated from TT emission and phosphorescence, versus extracted $\Delta E_{\mathrm{PIA}}$, revealing rough correlation between strength of binding and TT excited-state absorption. Dashed line is a guide to the eye with a slope of 1 .
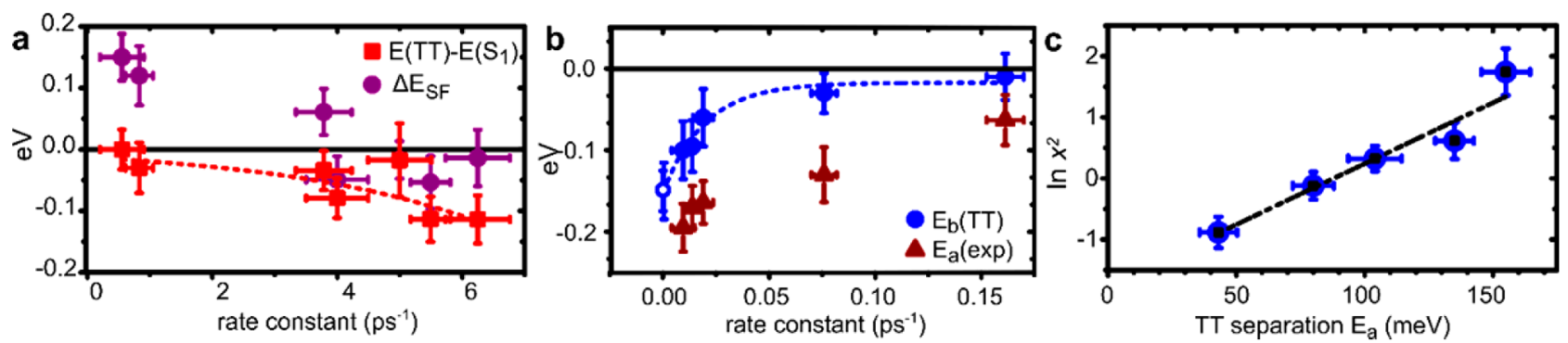

Figure 5. TT formation and dissociation rates. (a) $E(\mathrm{TT})-E\left(\mathrm{~S}_{1}\right)$ versus measured rate of SF. Standard SF exothermicity $\Delta E_{\mathrm{SF}}$ (circle) is not consistent with observed fission dynamics. Dashed curve is an exponential fit. (b) Trend in TT binding energy $E_{\mathrm{b}}(\mathrm{TT})$ (filled circle) is consistent with measured rates of TT separation, but activation energy $E_{\mathrm{a}}$ (triangle) extracted from temperature dependence is systematically larger. In Z-P and PF-M (empty circles), rates are estimated from exponential fit (dashed). (c) Proportionality of $E_{\mathrm{a}}$ for TT separation to nearest-neighbor separation parameter $\ln \left(x^{2}\right)$ reveals the process is governed by triplet hopping (details in Supporting Information). Error bars represent uncertainties in estimating the excited-state energy from the emission spectra and the exponential fitting of the rate constant and triplet lifetime.

phase. This phase periodically modulates the overall singlet character of the TT product state, with emission most likely near the composition immediately formed by SF (i.e., ${ }^{1}(\mathrm{TT})$ ). Whereas previously such PL quantum beating was attributed to triplet-triplet annihilation into fluorescent $S_{1}$ via ${ }^{1}(\mathrm{TT}),{ }^{15,30,53,54}$ that pathway is evidently unavailable here: we detect no delayed fluorescence in any film in the $S_{1}$ spectral region, suggesting this annihilation channel is energetically inaccessible from the observed TT state. Instead, we observe the beating directly in the TT emission, and we thereby link the ability of this state to emit to its time-variant overall singlet character.
Photoluminescence from this multiexcitonic state is surprising, as the transition should be one-photon forbidden. Following previous work in acenes, ${ }^{15}$ we invoke a HerzbergTeller mechanism in which TT couples vibronically to the bright $S_{1}$ state. This vibronic coupling effectively breaks the symmetry of the TT state, enabling intensity borrowing from the allowed $S_{1} \rightarrow S_{0}$ transition and imparting nonzero oscillator strength. This is the same mechanism by which the onephoton-forbidden $2 \mathrm{~A}_{\mathrm{g}}$ state is known to emit in carotenoids, ${ }^{55}$ and indeed, in some contexts the $2 \mathrm{~A}_{\mathrm{g}}$ state is also formally treated as a bound triplet pair. ${ }^{56}$ The prominent TT emission observed here compared to the acenes ${ }^{15}$ suggests that the Herzberg-Teller coupling mechanism is enhanced by diradical 
character. Under this model, the $0-0$ transition is suppressed or forbidden in emission and the most prominent peak is assigned to the $0-1$ band $\left[E\left(\mathrm{TT}_{0-1}\right)\right]$. Adding the energy of one TT vibronic spacing, we obtain $E(\mathrm{TT})_{0-0}$, the critical parameter for SF energetics (Table 1).

TT Binding Energy. The energies extracted from our optical measurements show that TT is a bound state, stabilized with respect to $T+T$ and $S_{1}$; this stabilization has been proposed in acenes to arise from wave function mixing with singlet and charge-transfer configurations. ${ }^{6,15,57} \mathrm{We}$ find that this directly affects the TT excited-state absorption spectrum, where triplet-like transitions $\left(\mathrm{T}_{1} \rightarrow \mathrm{T}_{n}, 800-1200 \mathrm{~nm}\right)$ demonstrate the presence of significant triplet character in the wave function. ${ }^{58}$ As noted above, in every molecule these features are blue shifted in TT relative to free or solutionsensitized triplets (Figure $4 a-c$ ). Throughout the series, the magnitude of the blue shift estimated from $\mathrm{T}+\mathrm{T}$ to $\mathrm{TT}$ peaks roughly follows the TT binding energy $\left(E_{\mathrm{b}}(\mathrm{TT})=E(\mathrm{TT})-\right.$ $E(T+T)$, Figure $4 d)$, suggesting these spectral shapes may help approximate the strength of $\mathrm{T}-\mathrm{T}$ interactions. From this correlation we infer that most of the spectral shift is due to stabilization of the " $\mathrm{T}_{1}$ " level within TT; in contrast, upper $\mathrm{T}_{n}$ is relatively unperturbed, and presumably in the " $\mathrm{T}_{1} \mathrm{~T}_{n}$ " state the triplets do not interact strongly. In Figure $5 \mathrm{a}$ and $5 \mathrm{~b}$ we plot TT formation and dissociation rates for all molecules at $298 \mathrm{~K}$. TT formation (i.e., SF) tracks with $E(\mathrm{TT})-E\left(\mathrm{~S}_{1}\right)$ rather than conventional $\Delta E_{\mathrm{SF}}{ }^{49}$ This effect is most evident in endothermic PF-M and Z-P, which can only undergo SF due to TT stabilization. Throughout the series we observe an enhancement in the SF rate with increasing driving force, a sign that these materials are not yet in the regime of excess exoergicity previously reported for heteroacenes in solution. ${ }^{49}$

During subsequent separation into $\mathrm{T}+\mathrm{T}$, we generally observe a slower rate for larger $\left|E_{\mathrm{b}}(\mathrm{TT})\right|$. However, our extracted binding energy is systematically smaller than the activation energy $\left(E_{\mathrm{a}}\right)$ obtained from fitting the temperaturedependent kinetics. This suggests another thermally activated mechanism contributes to $\mathrm{T}+\mathrm{T}$ formation, namely, triplet hopping. The discrepancy $\left|E_{\mathrm{a}}\right|-\left|E_{\mathrm{b}}(\mathrm{TT})\right|$ is greater in $\mathrm{OZ}$ than in $\mathrm{HZ}$, which exhibits closer molecular packing, and follows the trend of nearest-neighbor distances controlled by side groups $(-\mathrm{F}>-\mathrm{M}>-\mathrm{T}){ }^{35,38}$ Indeed, using reported nearest-neighbor distances $x$, we find that $E_{\mathrm{a}}$ is directly proportional to $\ln \left(x^{2}\right)$ (Figure 5c), consistent with simple triplet hopping ${ }^{52}$ (details in Supporting Information). We posit that $E_{\mathrm{b}}(\mathrm{TT})$ only governs formation of $\mathrm{T}+\mathrm{T}$ when the energy scale for triplet hopping is much smaller.

Influence of Diradical Character on SF Potential. The large observed $E_{\mathrm{a}}$ means triplet-pair separation can be entirely suppressed. We then observe intrinsic TT decay, obtaining similar 5-8 ns lifetimes across the family of materials (Figure $3 \mathrm{~b})$. Under the assumption of near quantitative $S_{1} \rightarrow \mathrm{TT}$ conversion and using the measured PL quantum yields, we can estimate the radiative and nonradiative decay rates of TT in the absence of separation into $\mathrm{T}+\mathrm{T}$ (Figure 6a). Both rates exhibit an apparent dependence on diradical character $y$, with TT emission particularly favored in more diradicaloid systems. The nonradiative decay channel can be attributed to geminate triplet-triplet annihilation, presumably to a vibrationally "hot" ground state given the absence of any further electronic signatures in PL or transient absorption as previously proposed in pentacene single crystals, ${ }^{59}$ polyenes, ${ }^{18}$ and acene heterodimers. ${ }^{60}$ While the nonradiative decay rate may be considered
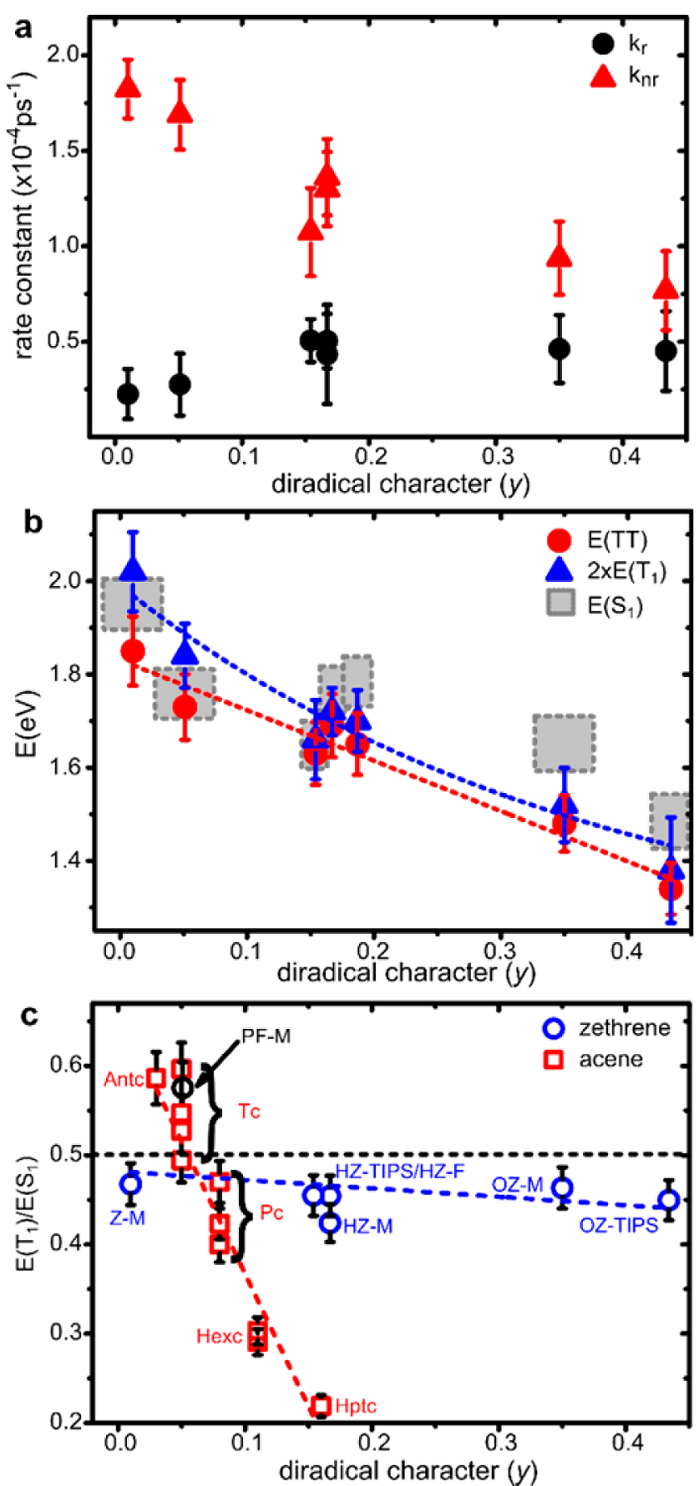

Figure 6. Effects of diradical character. (a) Rates of radiative $\left(k_{\mathrm{r}}\right)$ and nonradiative $\left(k_{\mathrm{nn}}\right)$ decay of TT at low temperature where no T+T is formed. The remarkably high observed $k_{\mathrm{r}}$ are $<10 \times$ smaller than $\mathrm{S}_{1}$ values in solution, and high diradical character particularly favors emission from TT. (b) Energies of states relevant to SF typically decrease with increasing diradical character. $E(\mathrm{TT})$ most closely tracks $E\left(\mathrm{~T}_{1}\right)$ rather than $E\left(\mathrm{~S}_{1}\right) . E(\mathrm{TT})$ decreases linearly with $y$ (red dashed), while $2 \times \mathrm{T}_{1}$ decreases exponentially (blue dashed). $y$ value for HZ-M has been shifted to 1.8 (from 1.679) for image clarity only. (c) Comparison of SF energetic condition $\left(E\left(T_{1}\right) / E\left(S_{1}\right) \leq 0.5\right)$ between acenes with 3 (Antc), 4 ( Tc), 5 (Pc), 6 (Hexc), and 7 (Hptc) fused rings and the reported zethrenes. In both classes, increasing the number of fused rings leads to greater diradical character. This results in little change in $E\left(\mathrm{~T}_{1}\right) / E\left(\mathrm{~S}_{1}\right)$ for zethrenes, but a precipitous dropoff in acenes which limits the range of chromophores suitable for SF applications $\left(E\left(\mathrm{~T}_{1}\right) / E\left(\mathrm{~S}_{1}\right) \approx 0.5\right)$. Dashed lines are a guide to the eye. Error bars correspond to uncertainties in determining the lifetime of TT (for rate constants) and the excited-state energy from emission spectra.

low relative to many intramolecular SF systems in which subnanosecond TT lifetimes are common, ${ }^{9,18,51,60-62}$ it appears to be high for a solid state system. It is difficult to directly compare these rates with other systems, as it is very uncommon to completely suppress the $\mathrm{TT} \rightarrow \mathrm{T}+\mathrm{T}$ process with 
temperature. However, the overall TT lifetimes in the zethrenes are shorter than found in acenes ${ }^{6}$ and heteroacenes ${ }^{15}$ even though TT separation remains active in the latter, and we infer that the zethrenes have relatively high nonradiative decay rates. Moreover, this decay channel is temperature independent in $\mathrm{Z}$ $\mathrm{P}$ and PF-M, suggesting an unconventional nonradiative decay mechanism, and we expect the same to be true of the other zethrenes as well, likely to be related to the diradical character. Given the ubiquity of rapid geminate annihilation following SF in other materials, $9,15,17,18,20,51,60,63-67$ this behavior calls for further investigation.

In Figure $6 \mathrm{~b}$ we plot measured $\mathrm{S}_{1}$, $\mathrm{TT}$, and $2 \times \mathrm{T}_{1}$ energies against $y$. These support earlier computational predictions, in particular, the onset of $E\left(S_{1}\right)>2 \times E\left(T_{1}\right)$ for $y \approx>0.1{ }^{33}$ Even at lower $y$, stabilization of the entangled triplet pair enables efficient, subpicosecond, temperature-independent SF. While $E(\mathrm{TT})$ most closely tracks $2 \times \mathrm{T}_{1}$, rather than $S_{1}$, the freetriplet energy is reduced much more considerably as $y$ increases. As a result, for moderate values of $y$ ( $\mathrm{HZ}$ and $\mathrm{OZ}) \mathrm{TT}$ separation occurs readily at room temperature. For smaller $y$, we observe more strongly bound TT states. Such materials may still be candidates for SF-sensitized solar cells: as recently demonstrated in films of $\mathrm{F}_{2}$-TES-ADT, it is possible to realize multielectron transfer directly from the bound pair. ${ }^{15}$

These diradicaloids thus constitute a versatile new SF materials platform both for fundamental study and for incorporation into solar cells. They maintain appropriate energetics over a wide range of $y$ parameters (Figure 6c), alongside tuning of the band gap over $1.4-1.9 \mathrm{eV}$. This tunability arises from the diradical resonance structures, in which increasing the molecular size simultaneously increases both the diradical character and the number of aromatic sextet rings. By contrast, acenes exhibit only one aromatic sextet regardless of the number of annealed rings and have electronic properties and reactivity that change rapidly with molecular size. ${ }^{31}$ Accordingly, a much wider range of zethrenes is suitable for SF, with additional benefits of high absorption coefficients ${ }^{47}$ and photo/chemical stability, ${ }^{35,68}$ demonstrating the power of diradical stabilization to generate next-generation SF materials.

\section{ASSOCIATED CONTENT}

\section{S Supporting Information}

The Supporting Information is available free of charge on the ACS Publications website at DOI: 10.1021/jacs.7b10762.

Details of materials, sample preparation, spectroscopic measurements, spectral decomposition, triplet energy characterization, transient-absorption spectroscopy in solution, room-temperature and temperature-dependent excited-state dynamics in thin film, characterization of pump-induced thermal effects, and calculation of triplet hopping activation energy (PDF)

\section{AUTHOR INFORMATION}

\section{Corresponding Authors}

*chmwuj@nus.edu.sg

*ncg11@cam.ac.uk

*a.musser@sheffield.ac.uk

\section{ORCID}

Jishan Wu: 0000-0002-8231-0437

Neil C. Greenham: 0000-0002-2155-2432

Andrew J. Musser: 0000-0002-4600-6606

\section{Notes}

The authors declare no competing financial interest. The data underlying this paper are available at https://doi.org/ 10.17863/CAM.15864.

\section{ACKNOWLEDGMENTS}

S.L. thanks AGS(O) Scholarship support from A*STAR Singapore. J.W. acknowledges financial support from MOE Tier 3 grant (MOE2014-T3-1-004). This work was supported by the Engineering and Physical Sciences Research Council, U.K. (Grant numbers EP/M005143/1, EP/G060738/1, and $\mathrm{EP} / \mathrm{M} 025330 / 1)$.

\section{REFERENCES}

(1) Smith, M. B.; Michl, J. Chem. Rev. 2010, 110, 6891-6936.

(2) Johnson, R. C.; Merrifield, R. E.; Avakian, P.; Flippen, R. B. Phys. Rev. Lett. 1967, 19, 285-287.

(3) Wilson, M. W. B.; Rao, A.; Clark, J.; Kumar, R. S. S.; Brida, D.; Cerullo, G.; Friend, R. H. J. Am. Chem. Soc. 2011, 133, 11830-11833.

(4) Musser, A. J.; Liebel, M.; Schnedermann, C.; Wende, T.; Kehoe, T. B.; Rao, A.; Kukura, P. Nat. Phys. 2015, 11, 352-357.

(5) Chan, W.-L.; Ligges, M.; Jailaubekov, A.; Kaake, L.; Miaja-Avila, L.; Zhu, X.-Y. Science 2011, 334, 1541-1545.

(6) Stern, H. L.; Cheminal, A.; Yost, S. R.; Broch, K.; Bayliss, S. L.; Chen, K.; Tabachnyk, M.; Thorley, K.; Greenham, N.; Hodgkiss, J. M.; Anthony, J.; Head-Gordon, M.; Musser, A. J.; Rao, A.; Friend, R. H. Nat. Chem. 2017, 9, 1205.

(7) Rao, A.; Wilson, M. W.; Albert-Seifried, S.; Di Pietro, R.; Friend, R. H. Phys. Rev. B: Condens. Matter Mater. Phys. 2011, 84, 195411.

(8) Wang, C.; Tauber, M. J. J. Am. Chem. Soc. 2010, 132, 1398813991.

(9) Sanders, S. N.; Kumarasamy, E.; Pun, A. B.; Trinh, M. T.; Choi, B.; Xia, J.; Taffet, E. J.; Low, J. Z.; Miller, J. R.; Roy, X.; Zhu, X. Y.; Steigerwald, M. L.; Sfeir, M. Y.; Campos, L. M. J. Am. Chem. Soc. 2015, 137, 8965-8972.

(10) Johnson, J. C.; Nozik, A. J.; Michl, J. J. Am. Chem. Soc. 2010, $132,16302-16303$.

(11) Hanna, M.; Nozik, A. J. Appl. Phys. 2006, 100, 074510.

(12) Ehrler, B.; Wilson, M. W. B.; Rao, A.; Friend, R. H.; Greenham, N. C. Nano Lett. 2012, 12, 1053-1057.

(13) Congreve, D. N.; Lee, J.; Thompson, N. J.; Hontz, E.; Yost, S. R.; Reusswig, P. D.; Bahlke, M. E.; Reineke, S.; Van Voorhis, T.; Baldo, M. A. Science 2013, 340, 334-337.

(14) Yang, L.; Tabachnyk, M.; Bayliss, S. L.; Böhm, M. L.; Broch, K.; Greenham, N. C.; Friend, R. H.; Ehrler, B. Nano Lett. 2015, 15, 354358.

(15) Yong, C. K.; Musser, A. J.; Bayliss, S. L.; Lukman, S.; Tamura, H.; Bubnova, O.; Hallani, R. K.; Meneau, A.; Resel, R.; Maruyama, M.; Hotta, S.; Herz, L. M.; Beljonne, D.; Anthony, J. E.; Clark, J.; Sirringhaus, H. Nat. Commun. 2017, 8, 15953.

(16) Austin, R. H.; Baker, G. L.; Etemad, S.; Thompson, R. J. Chem. Phys. 1989, 90, 6642-6646.

(17) Musser, A. J.; Al-Hashimi, M.; Maiuri, M.; Brida, D.; Heeney, M.; Cerullo, G.; Friend, R. H.; Clark, J. J. Am. Chem. Soc. 2013, 135, 12747-12754.

(18) Musser, A. J.; Maiuri, M.; Brida, D.; Cerullo, G.; Friend, R. H.; Clark, J. J. Am. Chem. Soc. 2015, 137, 5130-5139.

(19) Le, A. K.; Bender, J. A.; Roberts, S. T. J. Phys. Chem. Lett. 2016, 7, 4922-4928.

(20) Margulies, E. A.; Miller, C. E.; Wu, Y.; Ma, L.; Schatz, G. C.; Young, R. M.; Wasielewski, M. R. Nat. Chem. 2016, 8, 1120-1125.

(21) Hartnett, P. E.; Margulies, E. A.; Mauck, C. M.; Miller, S. A.; Wu, Y.; Wu, Y.-L.; Marks, T. J.; Wasielewski, M. R. J. Phys. Chem. B 2016, 120, 1357-1366.

(22) Hartnett, P. E.; Dyar, S. M.; Margulies, E. A.; Shoer, L. E.; Cook, A. W.; Eaton, S. W.; Marks, T. J.; Wasielewski, M. R. Chem. Sci. 2015, $6,402-411$ 
(23) Ryerson, J. L.; Schrauben, J. N.; Ferguson, A. J.; Sahoo, S. C.; Naumov, P. e.; Havlas, Z. k.; Michl, J.; Nozik, A. J.; Johnson, J. C. J. Phys. Chem. C 2014, 118, 12121-12132.

(24) Schrauben, J. N.; Ryerson, J. L.; Michl, J.; Johnson, J. C. J. Am. Chem. Soc. 2014, 136, 7363-7373.

(25) Walker, B. J.; Musser, A. J.; Beljonne, D.; Friend, R. H. Nat. Chem. 2013, 5, 1019-1024.

(26) Stern, H. L.; Musser, A. J.; Gelinas, S.; Parkinson, P.; Herz, L. M.; Bruzek, M. J.; Anthony, J.; Friend, R. H.; Walker, B. J. Proc. Natl. Acad. Sci. U. S. A. 2015, 112, 7656-7661.

(27) Tayebjee, M. J. Y.; Sanders, S. N.; Kumarasamy, E.; Campos, L. M.; Sfeir, M. Y.; McCamey, D. R. Nat. Phys. 2017, 13, 182-188.

(28) Weiss, L. R.; Bayliss, S. L.; Kraffert, F.; Thorley, K. J.; Anthony, J. E.; Bittl, R.; Friend, R. H.; Rao, A.; Greenham, N. C.; Behrends, J. Nat. Phys. 2017, 13, 176-181.

(29) Monahan, N. R.; Sun, D.; Tamura, H.; Williams, K. W.; Xu, B.; Zhong, Y.; Kumar, B.; Nuckolls, C.; Harutyunyan, A. R.; Chen, G.; Dai, H.-L.; Beljonne, D.; Rao, Y.; Zhu, X. Y. Nat. Chem. 2017, 9, 341346.

(30) Burdett, J. J.; Bardeen, C. J. J. Am. Chem. Soc. 2012, 134, 85978607

(31) Anthony, J. E. Angew. Chem., Int. Ed. 2008, 47, 452-483.

(32) Minami, T.; Ito, S.; Nakano, M. J. Phys. Chem. Lett. 2013, 4, $2133-2137$

(33) Minami, T.; Nakano, M. J. Phys. Chem. Lett. 2012, 3, 145-150. (34) Ito, S.; Minami, T.; Nakano, M. J. Phys. Chem. C 2012, 116, 19729-19736.

(35) Li, Y.; Heng, W.-K.; Lee, B. S.; Aratani, N.; Zafra, J. L.; Bao, N.; Lee, R.; Sung, Y. M.; Sun, Z.; Huang, K.-W.; Webster, R. D.; López Navarrete, J. T.; Kim, D.; Osuka, A.; Casado, J.; Ding, J.; Wu, J. J. Am. Chem. Soc. 2012, 134, 14913-14922.

(36) Umeda, R.; Hibi, D.; Miki, K.; Tobe, Y. Org. Lett. 2009, 11, 4104-4106.

(37) Wu, T.-C.; Chen, C.-H.; Hibi, D.; Shimizu, A.; Tobe, Y.; Wu, Y.T. Angew. Chem., Int. Ed. 2010, 49, 7059-7062.

(38) Hu, P.; Lee, S.; Park, K. H.; Das, S.; Herng, T. S.; Gonçalves, T. P.; Huang, K.-W.; Ding, J.; Kim, D.; Wu, J. J. Org. Chem. 2016, 81, 2911-2919.

(39) Hu, P.; Lee, S.; Herng, T. S.; Aratani, N.; Gonçalves, T. P.; Qi, Q.; Shi, X.; Yamada, H.; Huang, K.-W.; Ding, J.; Kim, D.; Wu, J. J. Am. Chem. Soc. 2016, 138, 1065-1077.

(40) Désilets, D.; Kazmaier, P. M.; Burt, R. A. Can. J. Chem. 1995, 73, 319-324.

(41) Hu, P.; Wu, J. Can. J. Chem. 2016, 95, 223-233.

(42) Becke, A. D. J. Chem. Phys. 1993, 98, 5648-5652.

(43) Lee, C.; Yang, W.; Parr, R. G. Phys. Rev. B: Condens. Matter Mater. Phys. 1988, 37, 785-789.

(44) Yanai, T.; Tew, D. P.; Handy, N. C. Chem. Phys. Lett. 2004, 393, $51-57$.

(45) Yamaguchi, K.; Fueno, T.; Fukutome, H. Chem. Phys. Lett. 1973, $22,461-465$.

(46) Nakano, M.; Champagne, B. Wiley Interdiscip. Rev. Comput. Mol. Sci. 2016, 6, 198-210.

(47) Huang, R.; Phan, H.; Herng, T. S.; Hu, P.; Zeng, W.; Dong, S.q.; Das, S.; Shen, Y.; Ding, J.; Casanova, D.; Wu, J. J. Am. Chem. Soc. 2016, 138, 10323-10330.

(48) Thompson, N. J.; Hontz, E.; Chang, W.; Van Voorhis, T.; Baldo, M. Philos. Trans. R. Soc., A 2015, 373, 20140323.

(49) Zhang, Y.-D.; Wu, Y.; Xu, Y.; Wang, Q.; Liu, K.; Chen, J.-W.; Cao, J.-J.; Zhang, C.; Fu, H.; Zhang, H.-L. J. Am. Chem. Soc. 2016, 138, 6739-6745.

(50) Di Motta, S.; Negri, F.; Fazzi, D.; Castiglioni, C.; Canesi, E. V. J. Phys. Chem. Lett. 2010, 1, 3334-3339.

(51) Lukman, S.; Chen, K.; Hodgkiss, J. M.; Turban, D. H. P.; Hine, N. D. M.; Dong, S.; Wu, J.; Greenham, N. C.; Musser, A. J. Nat. Commun. 2016, 7, 13622.

(52) Korovina, N. V.; Das, S.; Nett, Z.; Feng, X.; Joy, J.; Haiges, R.; Krylov, A. I.; Bradforth, S. E.; Thompson, M. E. J. Am. Chem. Soc. 2016, 138, 617-627.
(53) Wang, R.; Zhang, C.; Zhang, B.; Liu, Y.; Wang, X.; Xiao, M. Nat. Commun. 2015, 6, 8602.

(54) Chabr, M.; Wild, U. P.; Fünfschilling, J.; Zschokke-Gränacher, I. Chem. Phys. 1981, 57, 425-430.

(55) Christensen, R. L.; Kohler, B. E. J. Chem. Phys. 1975, 63, 18371846.

(56) Tavan, P.; Schulten, K. Phys. Rev. B: Condens. Matter Mater. Phys. 1987, 36, 4337-4358.

(57) Feng, X.; Luzanov, A. V.; Krylov, A. I. J. Phys. Chem. Lett. 2013, 4, 3845-3852.

(58) Chien, A. D.; Zimmerman, P. M. J. Chem. Phys. 2017, 146, 014103 .

(59) Poletayev, A. D.; Clark, J.; Wilson, M. W. B.; Rao, A.; Makino, Y.; Hotta, S.; Friend, R. H. Adv. Mater. 2014, 26, 919-924.

(60) Sanders, S. N.; Kumarasamy, E.; Pun, A. B.; Steigerwald, M. L.; Sfeir, M. Y.; Campos, L. M. Angew. Chem., Int. Ed. 2016, 55, 33733377.

(61) Lukman, S.; Musser, A. J.; Chen, K.; Athanasopoulos, S.; Yong, C. K.; Zeng, Z.; Ye, Q.; Chi, C.; Hodgkiss, J. M.; Wu, J. Adv. Funct. Mater. 2015, 25, 5452-5461.

(62) Sanders, S. N.; Kumarasamy, E.; Pun, A. B.; Appavoo, K.; Steigerwald, M. L.; Campos, L. M.; Sfeir, M. Y. J. Am. Chem. Soc. 2016 138, 7289-7297.

(63) Chang, H.-T.; Chang, Y.-Q.; Han, R.-M.; Wang, P.; Zhang, J.-P.; Skibsted, L. H. J. Agric. Food Chem. 2017, 65, 6058-6062.

(64) Busby, E.; Xia, J.; Wu, Q.; Low, J. Z.; Song, R.; Miller, J. R.; Zhu, X. Y.; Campos; Luis, M.; Sfeir, M. Y. Nat. Mater. 2015, 14, 426-433.

(65) Zirzlmeier, J.; Lehnherr, D.; Coto, P. B.; Chernick, E. T.; Casillas, R.; Basel, B. S.; Thoss, M.; Tykwinski, R. R.; Guldi, D. M. Proc. Natl. Acad. Sci. U. S. A. 2015, 112, 5325-5330.

(66) Kasai, Y.; Tamai, Y.; Ohkita, H.; Benten, H.; Ito, S. J. Am. Chem. Soc. 2015, 137, 15980-15983.

(67) Wang, C.; Angelella, M.; Kuo, C.-H.; Tauber, M. Proc. SPIE 2012, 8459, 845905-845905-13.

(68) Kaur, I.; Jia, W.; Kopreski, R. P.; Selvarasah, S.; Dokmeci, M. R.; Pramanik, C.; McGruer, N. E.; Miller, G. P. J. Am. Chem. Soc. 2008, 130, 16274-16286. 